\title{
A THEORY FOR THE LONGITUDINAL VIBRATIONS OF ANISOTROPIC ELASTIC BARS BASED ON THE RAYLEIGH-BISHOP MODEL
}

\author{
István ECSEDI AND ATtila BAKsA \\ Institute of Applied Mechanics, University of Miskolc \\ H-3515 Miskolc-Egyetemváros, Hungary \\ mechecs@uni-miskolc.hu, mechab@uni-miskolc.hu \\ [Received: February 11, 2019; Accepted: May 6, 2019]
}

\begin{abstract}
A Rayleigh-Bishop type theory of the longitudinal vibrations is developed for anisotropic bars. The equation of motion and the associated boundary conditions are derived by the use of Hamilton's principle. For an orthotropic bar the dependence of the eigenfrequencies from the position of the material coordinate system is analyzed. The numerical results obtained by the application of approximate analytical solution for the eigenfrequencies are compared with a FEM solution.
\end{abstract}

Mathematical Subject Classification: 74K10, 74H45, 74E10

Keywords: Longitudinal vibrations, bar, anisotropic, Rayleigh-Bishop theory.

\section{INTRODUCTION}

Modeling of vibration problems of elastic structural components is of great importance in engineering. The longitudinal vibration of thin isotropic elastic bar has been studied by many researchers over a long time owing to its wide applications in engineering [1 6]. The classical approximate theory of longitudinal vibration of isotropic bar was developed during the 18th century by J. D'Alambert, D. Bernoulli, L. Euler and J. Lagrange. This theory, which is called the simple theory of longitudinal vibration, is based on the analysis of the one-dimensional wave equation and is applicable for long and relatively thin bars vibrating at low frequency. The lateral effects and the corresponding lateral and axial shear modes are fully neglected in the frame of this simple theory of longitudinal vibration [1, 6]. J. Rayleigh [2] was the first who introduced the inertia of the lateral motions by which the cross sections are extended or contracted in their own planes. The lateral inertia effects are, however, important if the bar is thick. R. Bishop 7] further modified the Rayleigh theory in 1952 by taking into account the lateral shear effects. This theory is referred to as the Rayleigh-Bishop theory. From the point of view of the engineering applications this theory is slightly more complicated than the Rayleigh theory. The simple theory, the Rayleigh theory and Rayleigh-Bishop theory of longitudinal vibrations of isotropic bars are based on a fundamental assumption which says that the dynamics of the bar is described by a single unknown function and hence, a single partial differential equation containing the axial and time coordinates describes the longitudinal vibrations of isotropic 
elastic bars. Another important assumption of the above mentioned three theories is that the axial displacements do not depend on the cross-sectional coordinates, they depend only on the axial and time coordinates. The stress boundary conditions on the cylindrical boundary surface of the bar are fully ignored in these theories.

In a paper by Shatalov et al. [8] the simple theory, the Rayleigh, Rayleigh-Bishop, Herrmann and multimode models of the longitudinal vibrations of isotropic bars are compared with the exact Pochhammer-Chree solutions of the axisymmetric vibration of an isotropic cylinder. Application of two kinds of eigenfunction orthogonality in the Rayleigh and Rayleigh-Bishop theories of longitudinal vibrations of thick isotropic elastic bars is presented by Fedotov et al. [9], which deals with the analytical solution of vibration problem based on Green's function. A paper by Shatalov et al. [10] presents the multimode theories of the longitudinal vibration of isotropic elastic bars. The multimode models formulated in 10 can be considered as generalizations of the classical Rayleigh and Rayleigh-Bishop theories.

In this paper an approximate theory of the longitudinal vibration of anisotropic bars is presented. Equations of motion and boundary conditions are derived from Hamilton's principle 6, 11]. In the derived model the inertia of the lateral motions by which the cross-sections are extended or contracted in their own planes is considered. The contribution of more than one stiffness coefficients (elastic moduli) of the general anistropic material to the strain energy is introduced. From the assumed form of the displacement field a purely longitudinal (axial) vibration can be derived, so that the couplings between the longitudinal, torsional and flexural vibrations cannot be described in this approximate theory.

The connection between the position of material coordinate system, which is given by an angle coordinate, and the eigenfrequencies are analyzed for orthotropic bars. The numerical results obtained for the eigenfrequencies are checked by finite element computations.

\section{MECHANICAL MODEL}

The considered anisotropic elastic bar with uniform cross section is shown in Figure 1. The governing equations are formulated in the Cartesian coordinate system Oxyz. The position of the left end cross section is given by $z=0$, and the position of the right-end cross section is determined by $z=L$, where $L$ is the length of the bar. The $z$ axis is the centerline of the bar while the $x$ and $y$ axes are the principal axes of the left-end cross section. Hence we have

$$
\int_{A} x \mathrm{~d} A=\int_{A} y \mathrm{~d} A=0, \quad \int_{A} x y \mathrm{~d} A=0 .
$$

The material of the bar is a general anisotropic material referred to as triclinic material. In this case the generalized Hooke's law can be formulated as 12, 13.

$$
\sigma=C \varepsilon
$$

where

$$
\boldsymbol{\sigma}^{T}=\left[\begin{array}{llllll}
\sigma_{x} & \sigma_{y} & \sigma_{z} & \tau_{y z} & \tau_{x z} & \tau_{x y}
\end{array}\right], \quad \varepsilon^{T}=\left[\begin{array}{llllll}
\varepsilon_{x} & \varepsilon_{y} & \varepsilon_{z} & \gamma_{y z} & \gamma_{x z} & \gamma_{x y}
\end{array}\right],
$$



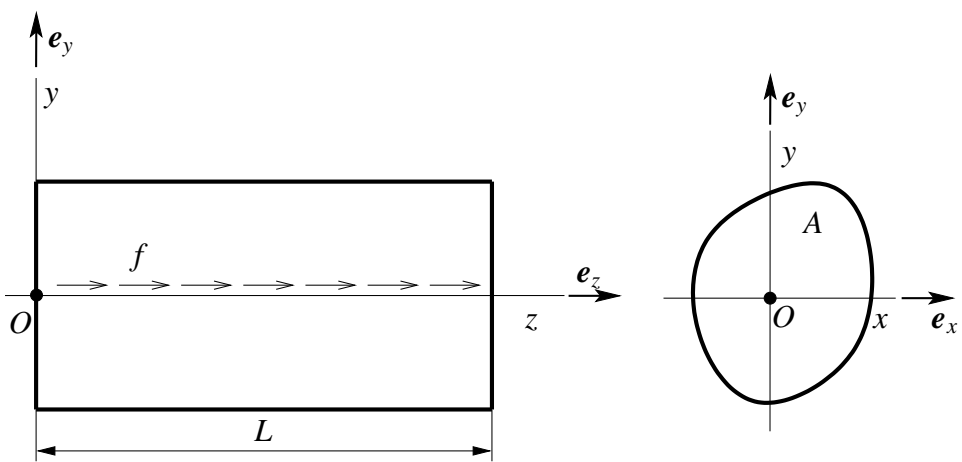

Figure 1. Anisotropic elastic bar with uniform cross section

$$
\boldsymbol{C}=\left[\begin{array}{llllll}
c_{11} & c_{12} & c_{13} & c_{14} & c_{15} & c_{16} \\
c_{12} & c_{22} & c_{23} & c_{24} & c_{25} & c_{26} \\
c_{13} & c_{23} & c_{33} & c_{34} & c_{35} & c_{36} \\
c_{14} & c_{24} & c_{34} & c_{44} & c_{45} & c_{46} \\
c_{15} & c_{25} & c_{35} & c_{45} & c_{55} & c_{56} \\
c_{16} & c_{26} & c_{36} & c_{46} & c_{56} & c_{66}
\end{array}\right]=\boldsymbol{C}^{T}
$$

while $\sigma_{x}, \sigma_{y}$ and $\sigma_{z}$ are the normal stresses, $\tau_{y z}, \tau_{x z}$ and $\tau_{x y}$ are the shear stresses, $\varepsilon_{x}, \varepsilon_{y}$ and $\varepsilon_{z}$ are the longitudinal strains, $\gamma_{y z}, \gamma_{x z}$ and $\gamma_{x y}$ are the shear strains, $c_{i j}$ $(i, j=1, \ldots, 6)$ are the elastic moduli of the considered anistropic elastic material. The compliance matrix $\boldsymbol{S}$ is the inverse of stiffness matrix $\boldsymbol{C}$ :

$$
\varepsilon=\boldsymbol{S} \boldsymbol{\sigma}, \quad \boldsymbol{S}=\boldsymbol{C}^{-1},
$$

where $\boldsymbol{S}=\left\{s_{i j}\right\}$ and $s_{i j}=s_{j i}(i, j=1, \ldots, 6)$.

Let the anisotropic bar be loaded at its end cross sections by uniform normal stresses, whose stress resultant is the axial force $P$. The displacement components associated with this axial force can be given in the following forms 12,13 :

$$
\begin{gathered}
u=\frac{P}{A}\left(s_{13} x+\frac{1}{2} s_{36} y\right), \\
v=\frac{P}{A}\left(\frac{1}{2} s_{36} x+s_{23} y\right), \\
w=\frac{P}{A}\left(s_{35} x+s_{34} y+s_{33} z\right)
\end{gathered}
$$

where $u, v$ and $w$ are the displacement components parallel to the axes $x, y$ and $z$; $A$ is the area of the bar cross section; $s_{13}, s_{23}, s_{33}, s_{34}, s_{35}$ and $s_{36}$ are the flexibility coefficients. It can be seen by substituting $P / A$ from the derivative of equation 2.6 with respect to $z$ into equations $(2.4)$ and 2.5 that

$$
u=\left(\frac{s_{13}}{s_{33}} x+\frac{1}{2} \frac{s_{36}}{s_{33}} y\right) \frac{\partial w}{\partial z},
$$




$$
v=\left(\frac{1}{2} \frac{s_{36}}{s_{33}} x+\frac{s_{23}}{s_{33}} y\right) \frac{\partial w}{\partial z} .
$$

Equations (2.7) and (2.8) give the lateral displacements in the anisotropic bar in terms of axial displacement $w$. In the sequel we shall assume that for longitudinal vibrations the displacements in the directions $x$ and $y$ directions are given by equations (2.7) and (2.8) and the axial displacement $w$ depends only on the axial coordinate $z$ and the time coordinate $t$. All results of this paper are based on the above-mentioned assumptions. The equation of motion and the boundary conditions are derived from Hamilton's principle, which can be stated as

$$
\delta \int_{t_{1}}^{t_{2}}(T-\Pi+W) \mathrm{d} t=0,
$$

where $T$ is the kinetic energy of the bar, $\Pi$ is the strain energy and the work done by the external force $f=f(z, t)$ is denoted by $W$ (Figure 1). Of all of the possible varied paths only those are considered that coincide with the true path at the two instants $t_{1}$ and $t_{2}$, 4, 6, 11.

First we determine the kinetic energy starting from the following equation

$$
\begin{aligned}
& T=\frac{1}{2} \int_{B} \rho\left[\left(\frac{\partial u}{\partial t}\right)^{2}+\left(\frac{\partial v}{\partial t}\right)^{2}+\left(\frac{\partial w}{\partial t}\right)^{2}\right] \mathrm{d} B \\
& =\frac{1}{2} \int_{B} \rho\left\{\left[\left(\frac{s_{13}}{s_{23}} x+\frac{1}{2} \frac{s_{36}}{s_{33}} y\right)^{2}+\left(\frac{1}{2} \frac{s_{36}}{s_{33}} x+\frac{s_{23}}{s_{33}} y\right)^{2}\right]\left(\frac{\partial^{2} w}{\partial z \partial t}\right)^{2}+\left(\frac{\partial w}{\partial t}\right)^{2}\right\} \mathrm{d} B .
\end{aligned}
$$

Here, $B$ is the space domain occupied by the cylindrical bar, $\rho$ is the mass density. A detailed computation, in which equation (2.1) has been used, yields

$$
T=\frac{1}{2} \int_{0}^{L}\left[A \rho\left(\frac{\partial w}{\partial t}\right)^{2}+\rho I\left(\frac{\partial^{2} w}{\partial z \partial t}\right)^{2}\right] \mathrm{d} z,
$$

where

$$
\begin{gathered}
I=\left[\left(\frac{s_{13}}{s_{33}}\right)^{2}+\frac{1}{4}\left(\frac{s_{36}}{s_{33}}\right)^{2}\right] I_{y}+\left[\frac{1}{4}\left(\frac{s_{36}}{s_{33}}\right)^{2}+\left(\frac{s_{23}}{s_{33}}\right)^{2}\right] I_{x} \\
=\left\{\left[\left(\frac{s_{13}}{s_{33}}\right)^{2}+\frac{1}{4}\left(\frac{s_{36}}{s_{33}}\right)^{2}\right] i_{y}^{2}+\left[\frac{1}{4}\left(\frac{s_{36}}{s_{33}}\right)^{2}+\left(\frac{s_{23}}{s_{33}}\right)^{2}\right] i_{x}^{2}\right\} A=i^{2} A \\
I_{x}=\int_{A} y^{2} \mathrm{~d} A=i_{x}^{2} A, \quad I_{y}=\int_{A} x^{2} \mathrm{~d} A=i_{y}^{2} A .
\end{gathered}
$$

For an anisotropic linearly elastic material the strain energy density as a function of strain field is obtained as 12,13 


$$
\begin{gathered}
a\left(\varepsilon_{x}, \varepsilon_{y}, \varepsilon_{z}, \gamma_{y z}, \gamma_{x z}, \gamma_{x y}\right)=\frac{1}{2}\left(c_{11} \varepsilon_{x}^{2}+2 c_{12} \varepsilon_{x} \varepsilon_{y}+2 c_{13} \varepsilon_{x} \varepsilon_{z}+2 c_{14} \varepsilon_{x} \gamma_{y z}+\right. \\
2 c_{15} \varepsilon_{x} \gamma_{x z}+2 c_{16} \varepsilon_{x} \gamma_{x y}+c_{22} \varepsilon_{y}^{2}+2 c_{23} \varepsilon_{y} \varepsilon_{z}+2 c_{24} \varepsilon_{y} \gamma_{y z}+2 c_{25} \varepsilon_{y} \gamma_{x z}+2 c_{26} \varepsilon_{y} \gamma_{x y} \\
+c_{33} \varepsilon_{z}^{2}+2 c_{34} \varepsilon_{z} \gamma_{y z}+2 c_{35} \varepsilon_{z} \gamma_{x z}+2 c_{36} \varepsilon_{z} \gamma_{x y} \\
\left.+c_{44} \gamma_{y z}^{2}+2 c_{45} \gamma_{y z} \gamma_{x z}+2 c_{46} \gamma_{y z} \gamma_{x y}+2 c_{55} \gamma_{x z}^{2}+2 c_{56} \gamma_{x z} \gamma_{x y}+c_{66} \gamma_{x y}^{2}\right)
\end{gathered}
$$

in which

$$
\begin{gathered}
\varepsilon_{x}=\frac{s_{13}}{s_{33}} \frac{\partial w}{\partial z}, \quad \varepsilon_{y}=\frac{s_{23}}{s_{33}} \frac{\partial w}{\partial z}, \quad \varepsilon_{z}=\frac{\partial w}{\partial z}, \\
\gamma_{y z}=\left(\frac{1}{2} \frac{s_{36}}{s_{33}} x+\frac{s_{23}}{s_{33}} y\right) \frac{\partial^{2} w}{\partial z^{2}}, \quad \gamma_{x z}=\left(\frac{s_{13}}{s_{33}} x+\frac{1}{2} \frac{s_{36}}{s_{33}} y\right) \frac{\partial^{2} w}{\partial z^{2}}, \quad \gamma_{x y}=\frac{s_{36}}{s_{33}} \frac{\partial w}{\partial z} .
\end{gathered}
$$

The whole strain energy of the anistropic cylindrical bar is calculated from the following equation

$$
\Pi=\int_{B} a\left(\varepsilon_{x}, \varepsilon_{y}, \varepsilon_{z}, \gamma_{y z}, \gamma_{x z}, \gamma_{x y}\right) \mathrm{d} B .
$$

A detailed calculation in which equation 2.1 is also used leads to the result

$$
\Pi=\frac{1}{2} \int_{0}^{L}\left[C_{1}\left(\frac{\partial w}{\partial z}\right)^{2}+C_{2}\left(\frac{\partial^{2} w}{\partial z^{2}}\right)^{2}\right] \mathrm{d} z,
$$

where

$$
\begin{gathered}
C_{1}=\left[c_{11}\left(\frac{s_{13}}{s_{33}}\right)^{2}+c_{22}\left(\frac{s_{23}}{s_{33}}\right)^{2}+c_{33}+c_{66}\left(\frac{s_{36}}{s_{33}}\right)^{2}\right. \\
\left.+2 c_{12} \frac{s_{13} s_{23}}{s_{33}^{2}}+2 c_{13} \frac{s_{13}}{s_{33}}+2 c_{23} \frac{s_{23}}{s_{33}}+2 c_{16} \frac{s_{13} s_{36}}{s_{33}^{2}}+2 c_{26} \frac{s_{23} s_{36}}{s_{33}^{2}}+2 c_{36} \frac{s_{36}}{s_{33}}\right] A \\
=\left[\frac{c_{11}}{c_{33}}\left(\frac{s_{13}}{s_{33}}\right)^{2}+\frac{c_{22}}{c_{33}}\left(\frac{s_{23}}{s_{33}}\right)^{2}+1+\frac{c_{66}}{c_{33}}\left(\frac{s_{36}}{s_{33}}\right)^{2}+2 \frac{c_{12}}{c_{33}} \frac{s_{13} s_{23}}{s_{33}^{2}}+\right. \\
\left.2 \frac{c_{13}}{c_{33}} \frac{s_{13}}{s_{33}}+2 \frac{c_{23}}{c_{33}} \frac{s_{23}}{s_{33}}+2 \frac{c_{16}}{c_{33}} \frac{s_{13} s_{36}}{s_{33}^{2}}+2 \frac{c_{26}}{c_{33}} \frac{s_{23} s_{36}}{s_{33}^{2}}+2 \frac{c_{36}}{c_{33}} \frac{s_{36}}{s_{33}}\right] c_{33} A=c_{1} c_{33} A, \\
+\left[\frac{1}{4} c_{44}\left(\frac{s_{36}}{s_{33}}\right)^{2}+c_{45} \frac{s_{13} s_{36}}{s_{33}^{2}}+c_{55}\left(\frac{s_{13}}{s_{33}}\right)^{2}\right] I_{y} \\
\left.=\left\{\frac{1}{4} \frac{c_{44}}{c_{33}}\left(\frac{s_{23}}{s_{33}}\right)^{2}+c_{45} \frac{s_{23}}{s_{33}}\right)^{2}+\frac{s_{36}}{s_{33}}+c_{55} \frac{1}{4}\left(\frac{s_{13} s_{36}}{s_{33}^{2}}+\frac{c_{55}}{s_{33}}\right)^{2}\right] I_{x} \\
\left.+\left[\frac{s_{13}}{s_{33}}\right)^{2}\right] i_{y}^{2} \\
\left.\left.\left(\frac{c_{23}}{s_{33}}\right)^{2}+\frac{c_{45}}{c_{33}} \frac{s_{23} s_{36}}{s_{33}^{2}}+\frac{1}{4} \frac{c_{55}}{c_{33}}\left(\frac{s_{36}}{s_{33}}\right)^{2}\right] i_{x}^{2}\right\} c_{33} A=c_{2} c_{33} A .
\end{gathered}
$$


The work $W$ done by the external force $f=f(z, t)$ on the axial displacement $w=w(z, t)$ is as follows

$$
W=\int_{0}^{L} f(z, t) w(z, t) \mathrm{d} z .
$$

The substitution of equations 2.11), 2.18) and 2.21 into equation (2.9) yields the equation of motion and the associated boundary conditions:

$$
\begin{gathered}
C_{2} \frac{\partial^{4} w}{\partial z^{4}}-\rho I \frac{\partial^{4} w}{\partial z^{2} \partial t^{2}}-C_{1} \frac{\partial^{2} w}{\partial z^{2}}+\rho A \frac{\partial^{2} w}{\partial t^{2}}=f \\
{\left.\left[C_{1} \frac{\partial w}{\partial z}+\rho I \frac{\partial^{3} w}{\partial z \partial t^{2}}-C_{2} \frac{\partial^{3} w}{\partial z^{3}}\right] \delta w\right|_{0} ^{L}+\left.\left(C_{2} \frac{\partial^{2} w}{\partial z^{2}}\right) \delta\left(\frac{\partial w}{\partial z}\right)\right|_{0} ^{L}=0}
\end{gathered}
$$

It can be seen from equations 2.7 and 2.8 that

$$
w=0, \quad \frac{\partial w}{\partial z}=0
$$

on an end cross section if it is rigidly fixed. Hence it holds there that

$$
\delta w=0, \quad \delta\left(\frac{\partial w}{\partial z}\right)=0 .
$$

Since $\delta w$, and $\delta\left(\frac{\partial w}{\partial z}\right)$ are arbitrary on free end cross sections it follows that the stress boundary conditions in terms of $w=w(z, t)$ on these cross sections assume the form

$$
\begin{gathered}
C_{1} \frac{\partial w}{\partial z}+\rho I \frac{\partial^{3} w}{\partial z \partial t^{2}}-C_{2} \frac{\partial^{3} w}{\partial z^{3}}=0 \\
C_{2} \frac{\partial^{2} w}{\partial z^{2}}=0
\end{gathered}
$$

\section{Free vibrations}

For free vibrations

$$
w(z, t)=W(z) \cos \omega t, \quad f=0
$$

is the solution of 2.22 in which $W(z)$ is the amplitude of motion and $\omega$ is the circular frequency of the vibrations. Substitution of solution (3.1) into equation 2.22) yields

$$
C_{2} \frac{\mathrm{d}^{4} W}{\mathrm{~d} z^{4}}+\left(\omega^{2} \rho I-C_{1}\right) \frac{\mathrm{d}^{2} W}{\mathrm{~d} z^{2}}-\omega^{2} \rho A W=0
$$

The homogeneous boundary conditions equation 3.2 is associated with are

$$
W=0, \quad \frac{\mathrm{d} W}{\mathrm{~d} z}=0
$$

for fixed-end cross sections,

$$
C_{1} \frac{\mathrm{d} W}{\mathrm{~d} z}-\omega^{2} \rho I \frac{\mathrm{d} W}{\mathrm{~d} z}-C_{2} \frac{\mathrm{d}^{3} W}{\mathrm{~d} z^{3}}=0, \quad \frac{\mathrm{d}^{2} W}{\mathrm{~d} z^{2}}=0
$$

for free-end cross section and

$$
W=0, \quad \frac{\mathrm{d}^{2} W}{\mathrm{~d} z^{2}}=0
$$


for loosely fixed end cross sections 6

The general solution of equation (3.2), which contains four integration constants, can be written in the form 14,15

$$
W(z)=K_{1} \cosh q z+K_{2} \sinh q z+K_{3} \cos r z+K_{4} \sin r z,
$$

where the constants $K_{1}, K_{2}, K_{3}$ and $K_{4}$ to be determined from the boundary conditions of the anistropic bar are such that one of them may have arbitrary value. As regards the parameters $q$ and $r$ we have

$$
\begin{gathered}
q=\sqrt{\frac{C_{1}-\omega^{2} \rho I+\sqrt{\left(C_{1}-\omega^{2} \rho I\right)^{2}+4 \omega^{2} C_{2} \rho A}}{2 C_{2}}}, \\
r=\sqrt{\frac{\sqrt{\left(C_{1}-\omega^{2} \rho I\right)^{2}+4 \omega^{2} C_{2} \rho A}-C_{1}+\omega^{2} \rho I}{2 C_{2}}} .
\end{gathered}
$$

As regards the free bending vibrations of an isotropic Euler-Bernoulli-Rayleigh beam subjected to an axial force, the differential equation and the boundary conditions associated with it are similar to those which describe the longitudinal free vibrations of the anisotropic bar considered in this paper. The differential equation and associated boundary condition for the bending vibrations of an isotropic EulerBernoulli beam with constant axial force are as follows [16:

- differential equation:

$$
E I_{x} \frac{\mathrm{d}^{4} V}{\mathrm{~d} z^{4}}+\left(\omega^{2} \rho J-P\right) \frac{\mathrm{d}^{2} V}{\mathrm{~d} z^{2}}-\omega^{2} \rho A V=0,
$$

- boundary conditions for fixed-end cross sections:

$$
V=0, \quad \frac{\mathrm{d} V}{\mathrm{~d} z}=0,
$$

- boundary conditions for free-end cross sections:

$$
P \frac{\mathrm{d} V}{\mathrm{~d} z}-E I_{x} \frac{\mathrm{d}^{3} V}{\mathrm{~d} z^{3}}-\rho \omega^{2} J \frac{\mathrm{d} V}{\mathrm{~d} z}=0, \quad \frac{\mathrm{d}^{2} V}{\mathrm{~d} z^{2}}=0,
$$

- boundary conditions for simply supported end cross sections:

$$
V=0, \quad \frac{\mathrm{d}^{2} V}{\mathrm{~d} z^{2}}=0 .
$$

In equations (3.9)-3.12) $V=V(z)$ is the amplitude of the displacement of centerline in the direction of axes $y, E$ is the Young modulus, $J=I_{x}+I_{y}, P>0$ is the applied axial force. The form of equations $3.9-(3.12)$ makes it possible to establish an analogy between the longitudinal vibration of anisotropic bar and the bending vibration of the isotropic Euler-Bernoulli-Rayleigh beam subjected to constant positive axial force. The analogy is shown in Table 1. 
Table 1. Analogous quantities and boundary conditions.

\begin{tabular}{|c|c|}
\hline $\begin{array}{c}\text { Longitudinal vibrations } \\
\text { of anisotropic bars }\end{array}$ & $\begin{array}{l}\text { Bending vibrations } \\
\text { of isotropic beams }\end{array}$ \\
\hline $\begin{array}{c}W=W(z) \\
C_{2} \\
C_{1} \\
I\end{array}$ & $\begin{array}{c}V=V(z) \\
E I_{x} \\
P>0 \\
J\end{array}$ \\
\hline Boundary conditions & Boundary conditions \\
\hline $\begin{array}{l}\text { fixed end: } \\
\qquad W=0, \frac{\mathrm{d} W}{\mathrm{~d} z}=0 \\
\text { free end: } \\
\left(C_{1}-\omega^{2} \rho I\right) \frac{\mathrm{d} W}{\mathrm{~d} z}-C_{2} \frac{\mathrm{d}^{3} W}{\mathrm{~d} z^{3}}=0, \frac{\mathrm{d}^{2} W}{\mathrm{~d} z^{2}}=0 \\
\text { loosely fixed end: } \\
\qquad W=0, \frac{\mathrm{d}^{2} W}{\mathrm{~d} z^{2}}=0\end{array}$ & $\begin{array}{l}\text { fixed end: } \\
\qquad V=0, \frac{\mathrm{d} V}{\mathrm{~d} z}=0 \\
\text { free end: } \\
\left(P-\omega^{2} \rho J\right) \frac{\mathrm{d} V}{\mathrm{~d} z}-E I_{x} \frac{\mathrm{d}^{3} V}{\mathrm{~d} z^{3}}=0, \frac{\mathrm{d}^{2} V}{\mathrm{~d} z^{2}}=0 \\
\text { simply supported end: } \\
\qquad V=0, \frac{\mathrm{d}^{2} V}{\mathrm{~d} z^{2}}=0\end{array}$ \\
\hline
\end{tabular}

\section{EXAMPLE}

Assume that the anisotropic bar is fixed loosely at both ends. Then the axial displacement and shear strains will be zero at each end of the bar. Hence

$$
W=0, \quad \frac{\mathrm{d}^{2} W}{\mathrm{~d} z^{2}}=0, \quad \text { at } \quad z=0 \quad \text { and } \quad z=L .
$$

It is obvious from taking the analogy formulated in Table 1 into account that the boundary conditions related to the loosely fixed end condition of the anisotropic bar are similar to those valid for the simply supported end conditions of the vibrating Euler-Bernoulli-Rayleigh beam. On the basis of this similarity we shall assume

$$
W_{j}(z)=W_{j} \sin j \frac{\pi}{L} z, \quad j=1,2, \ldots
$$

The function given by equation 4.2 satisfies the boundary conditions for any value of $W_{j}(j=1,2, \ldots)$

$$
W(0)=W(L)=0,\left.\quad \frac{\mathrm{d}^{2} W}{\mathrm{~d} z^{2}}\right|_{z=0}=\left.\frac{\mathrm{d}^{2} W}{\mathrm{~d} z^{2}}\right|_{z=L}=0 .
$$

From equation (3.2) it follows that the natural frequencies of the longitudinal vibration of anisotropic bar fixed loosely at both ends are expressed as

$$
\omega_{j}^{2}=\frac{C_{2}\left(\frac{j \pi}{L}\right)^{4}+C_{1}\left(\frac{j \pi}{L}\right)^{2}}{\rho\left(\frac{j \pi}{L}\right)^{2} I+A \rho}=\frac{c_{2}\left(\frac{j \pi}{L}\right)^{2}+c_{1}}{i^{2}\left(\frac{j \pi}{L}\right)^{2}+1} \Omega_{j}^{2}, \quad \Omega_{j}=\sqrt{\frac{c_{33}}{\rho}} \frac{j \pi}{L} \quad j=1,2, \ldots .
$$

In equation (4.4) $\Omega_{j}(j=1,2,3, \ldots)$ is the natural frequency of the longitudinal vibration of the considered bar with fixed ends, which can be derived by the application of the following assumptions (simple theory): 
- The lateral displacement $u$ and $v$ are neglected and $w=w(z, t)$.

- The dominant element of the stiffness matrix $\boldsymbol{C}$ is $c_{33}$, so the expression of axial force $N$ can be written in the form

$$
N=A c_{33} \frac{\partial w}{\partial z}
$$

This formulation does not introduce the effect of lateral motions of cross sections and the material anisotropy. The eigenfunction (mode shape) corresponding to the frequency $\omega_{j}$ is given by equation 4.2 .

\section{Dependence of the natural frequencies on the position of the PRINCIPAL MATERIAL COORDINATE SYSTEM}

We consider an orthotropic material whose principal directions are $x_{1}, x_{2}$ and $x_{3}=z\left[12,13,17,18\right.$. The position of the material coordinate system $O x_{1} x_{2} x_{3}$ is given by angle $\alpha$ as shown in Figure 2 17, 18 .

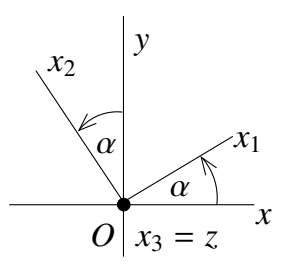

Figure 2. Definition of $\alpha$

For orthotropic elastic material in terms of engineerging constants $E_{1}, E_{2}, E_{3}, G_{23}$, $G_{13}, G_{12}, \nu_{12}, \nu_{21}, \nu_{13}, \nu_{31}, \nu_{23}, \nu_{32}$ the stiffness matrix $\boldsymbol{C}\left(x_{1}, x_{2}, x_{3}\right)$ can be written in the form [17, 18]

$$
\begin{aligned}
\boldsymbol{C}\left(x_{1}, x_{2}, x_{3}\right)= & {\left[\begin{array}{cccccc}
C_{11} & C_{12} & C_{13} & 0 & 0 & 0 \\
C_{12} & C_{22} & C_{23} & 0 & 0 & 0 \\
C_{13} & C_{23} & C_{33} & 0 & 0 & 0 \\
0 & 0 & 0 & C_{44} & 0 & 0 \\
0 & 0 & 0 & 0 & C_{55} & 0 \\
0 & 0 & 0 & 0 & 0 & C_{66}
\end{array}\right]=} \\
& {\left[\begin{array}{ccccccc}
\frac{1-\nu_{23} \nu_{32}}{E_{2} E_{3} \Delta} & \frac{\nu_{21}+\nu_{23} \nu_{31}}{E_{2} E_{3} \Delta} & \frac{\nu_{31}+\nu_{21} \nu_{32}}{E_{2} E_{3} \Delta} & 0 & 0 & 0 \\
\frac{\nu_{21}+\nu_{23} \nu_{31}}{E_{2} E_{3} \Delta} & \frac{1-\nu_{13} \nu_{31}}{E_{1} E_{3} \Delta} & \frac{\nu_{32}+\nu_{12} \nu_{31}}{E_{1} E_{3} \Delta} & 0 & 0 & 0 \\
\frac{\nu_{31}+\nu_{21} \nu_{32}}{E_{2} E_{3} \Delta} & \frac{\nu_{32}+\nu_{12} \nu_{31}}{E_{1} E_{3} \Delta} & \frac{1-\nu_{12} \nu_{21}}{E_{1} E_{2} \Delta} & 0 & 0 & 0 \\
0 & 0 & 0 & G_{23} & 0 & 0 \\
0 & 0 & 0 & 0 & G_{13} & 0 \\
0 & 0 & 0 & 0 & 0 & 0 & G_{12}
\end{array}\right], }
\end{aligned}
$$

where

$$
E_{1} \nu_{21}=E_{2} \nu_{12}, \quad E_{2} \nu_{32}=E_{3} \nu_{23}, \quad E_{1} \nu_{31}=E_{3} \nu_{13},
$$


and

$$
\Delta=\frac{1-\nu_{12} \nu_{21}-\nu_{23} \nu_{32}-\nu_{13} \nu_{31}-2 \nu_{21} \nu_{32} \nu_{13}}{E_{1} E_{2} E_{3}} .
$$

The inverse of $\boldsymbol{C}\left(x_{1}, x_{2}, x_{3}\right)$ gives the flexibility matrix $\boldsymbol{S}\left(x_{1}, x_{2}, x_{3}\right)$ defined in the principal material coordinate system $O x_{1} x_{2} x_{3}[17,18$ :

$$
\begin{aligned}
& \boldsymbol{S}\left(x_{1}, x_{2}, x_{3}\right)=\left[\begin{array}{cccccc}
S_{11} & S_{12} & S_{13} & 0 & 0 & 0 \\
S_{12} & S_{22} & S_{23} & 0 & 0 & 0 \\
S_{13} & S_{23} & S_{33} & 0 & 0 & 0 \\
0 & 0 & 0 & S_{44} & 0 & 0 \\
0 & 0 & 0 & 0 & S_{55} & 0 \\
0 & 0 & 0 & 0 & 0 & S_{66}
\end{array}\right]= \\
& {\left[\begin{array}{ccccccc}
\frac{1}{E_{1}} & -\frac{\nu_{21}}{E_{2}} & -\frac{\nu_{31}}{E_{3}} & 0 & 0 & 0 \\
-\frac{\nu_{12}}{E_{1}} & \frac{1}{E_{2}} & -\frac{\nu_{32}}{E_{3}} & 0 & 0 & 0 \\
-\frac{\nu_{13}}{E_{1}} & -\frac{\nu_{23}}{E_{2}} & \frac{1}{E_{3}} & 0 & 0 & 0 \\
0 & 0 & 0 & \frac{1}{G_{23}} & 0 & 0 \\
0 & 0 & 0 & 0 & \frac{1}{G_{13}} & 0 \\
0 & 0 & 0 & 0 & 0 & \frac{1}{G_{12}}
\end{array}\right] . }
\end{aligned}
$$

By the use of transformation stiffness and compliance matrices 17, 18 we can derive the formulas of $c_{i j}$ in terms of $C_{i j}$ and the formulas of $s_{i j}$ in terms of $S_{i j}$ :

$$
\begin{gathered}
c_{11}=m^{4} C_{11}+2 m^{2} n^{2}\left(C_{12}+2 C_{66}\right)+n^{4} C_{22}, \\
c_{12}=m^{2} n^{2}\left(C_{11}+C_{22}-4 C_{66}\right)+\left(m^{4}+n^{4}\right) C_{12}, \\
c_{13}=m^{2} C_{13}+n^{2} C_{23}, \\
c_{16}=m n\left[m^{2}\left(C_{11}-C_{12}-2 C_{66}\right)+n^{2}\left(C_{12}-C_{22}+2 C_{66}\right)\right], \\
c_{22}=n^{4} C_{11}+2 m^{2} n^{2}\left(C_{12}+2 C_{66}\right)+m^{4} C_{22}, \\
c_{23}=n^{2} C_{13}+m^{2} C_{23}, \\
c_{26}=m n\left[n^{2}\left(C_{11}-C_{12}-2 C_{66}\right)+m^{2}\left(C_{12}-C_{22}+2 C_{66}\right)\right], \\
c_{33}=C_{33}, \\
c_{36}=m n\left(C_{13}-C_{23}\right), \\
c_{44}=m^{2} C_{44}+n^{2} C_{55}, \\
c_{45}=m n\left(C_{55}-C_{44}\right), \\
c_{55}=n^{2} C_{44}+m^{2} C_{55}, \\
c_{66}=m^{2} n^{2}\left(C_{11}-2 C_{12}+C_{22}\right)+\left(n^{2}-m^{2}\right)^{2} C_{66} \\
c_{14}=c_{15}=c_{24}=c_{25}=c_{34}=c_{35}=c_{46}=c_{56}=0, \\
s_{11}=m^{4} S_{11}+m^{2} n^{2}\left(2 S_{12}+S_{66}\right)+n^{2} S_{22}, \\
s_{12}=m^{2} n^{2}\left(S_{11}+S_{22}-S_{66}\right)+\left(m^{4}+n^{4}\right) S_{12}, \\
s_{13}=m^{2} S_{13}+n^{2} S_{23}, \\
m n\left[m^{2}\left(2 S_{11}-2 S_{12}-S_{66}\right)+n^{2}\left(2 S_{12}-2 S_{22}+S_{66}\right)\right],
\end{gathered}
$$




$$
\begin{gathered}
s_{22}=n^{4} S_{11}+m^{2} n^{2}\left(2 S_{12}+S_{66}\right)+m^{4} S_{22}, \\
s_{23}=n^{2} S_{13}+m^{2} S_{23}, \\
s_{26}=m n\left[n^{2}\left(2 S_{11}-2 S_{12}-S_{66}\right)+m^{2}\left(2 S_{12}-2 S_{22}+S_{66}\right)\right], \\
s_{33}=S_{33}, \\
s_{36}=2 m n\left(S_{13}-S_{23}\right), \\
s_{44}=m^{2} S_{44}+n^{2} S_{55}, \\
s_{45}=m n\left(S_{55}-S_{44}\right), \\
s_{55}=n^{2} S_{44}+m^{2} S_{55}, \\
s_{66}=4 m^{2} n^{2}\left(S_{11}-2 S_{12}+S_{22}\right)+\left(n^{2}-m^{2}\right) S_{66}, \\
s_{14}=s_{15}=s_{24}=s_{25}=s_{34}=s_{35}=s_{46}=s_{56}=0, \\
m=\cos \alpha, \quad n=\sin \alpha .
\end{gathered}
$$

The dependence of the natural frequencies on $\alpha$ given by equation 4.4 for an orthotropic bar of rectangular cross section is analyzed by using the following data (Figure 3):

$$
\begin{gathered}
b_{1}=0.03 \mathrm{~m}, \quad b_{2}=0.05 \mathrm{~m}, \quad L=1 \mathrm{~m}, \quad \rho=550 \frac{\mathrm{kg}}{\mathrm{m}^{3}}, \\
E_{1}=11 \cdot 10^{8} \mathrm{~Pa}, \quad E_{2}=5.7 \cdot 10^{8} \mathrm{~Pa}, \quad E_{3}=163 \cdot 10^{8} \mathrm{~Pa} \\
G_{23}=11.06 \cdot 10^{8} \mathrm{~Pa}, \quad G_{13}=6.8 \cdot 10^{8} \mathrm{~Pa}, \quad G_{12}=0.66 \mathrm{~Pa}, \\
\nu_{12}=0.68, \quad \nu_{13}=0.038, \quad \nu_{23}=0.015
\end{gathered}
$$

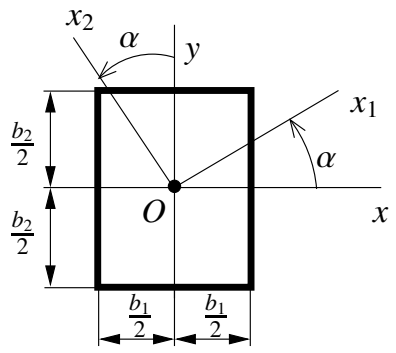

Figure 3. Solid rectangular cross section

In the present problem $\Omega_{1}=17102.61 / \mathrm{s}, \Omega_{2}=34205.21 / \mathrm{s}, \Omega_{3}=51307.81 / \mathrm{s}$, $\Omega_{4}=68410.41 / \mathrm{s}$, which do not depend on the size or the shape of the cross section. The graphs of $\omega_{j}(\alpha)$ and $\omega_{j}(\alpha) / \Omega_{j}$ for $j=1,2,3,4$ and $0 \leq \alpha \leq \pi$ are shown in Figure 4 . 

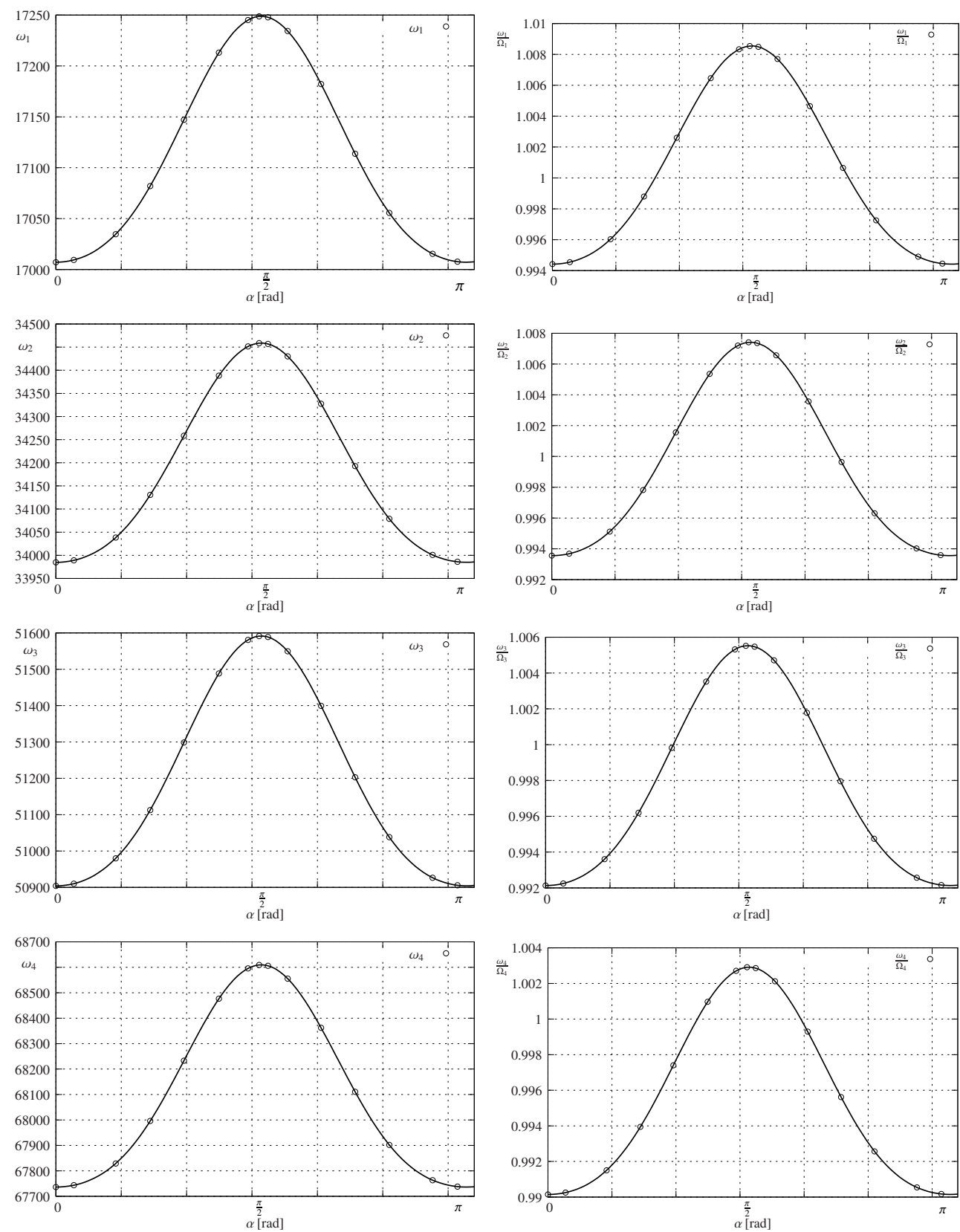

Figure 4. $\alpha$ dependence of the natural frequencies for $j=1,2,3,4$ 
In Table 2 the $\alpha$ dependence of the fundamental frequency obtained from equation 4.4 is compared to the results of FEM computations for $\alpha=0, \frac{\pi}{8}, \frac{\pi}{6}, \frac{\pi}{4}, \frac{\pi}{3}, \frac{\pi}{2}$.

Table 2. Comparison of analytical and FEM (Abaqus) solutions

\begin{tabular}{|c|c|c|}
\hline \multicolumn{3}{|c|}{$\omega_{1}(\alpha)\left[\frac{\mathrm{rad}}{\mathrm{s}}\right]$} \\
\hline \hline$\alpha$ & Analytical Solution & FEM Computation \\
\hline 0 & 17007.13 & 17094 \\
$\pi / 8$ & 17026.93 & 17094 \\
$\pi / 6$ & 17044.19 & 17094 \\
$\pi / 4$ & 17096.92 & 17093 \\
$\pi / 3$ & 17165.18 & 17093 \\
$\pi / 2$ & 17248.79 & 17092 \\
\hline
\end{tabular}

The results of analytical solution and FEM computations for the first four natural frequencies for $\alpha=0$ are listed in Table 3 .

Table 3. Comparison of analytical and FEM (Abaqus) solutions for $\alpha=0$

\begin{tabular}{|c|c|c|}
\hline \multicolumn{3}{|c|}{$\omega_{j}(0)\left[\frac{\mathrm{rad}}{\mathrm{s}}\right]$} \\
\hline \hline$j$ & Analytical Solution & FEM Computation \\
\hline 1 & 17007.13 & 17094 \\
2 & 33988.48 & 34130 \\
3 & 50904.12 & 50912 \\
4 & 67736.28 & 68191 \\
\hline
\end{tabular}

The results obtained from FEM computations support the validity of the presented approximate analytical method.

\section{Conclusions}

In this paper an approximate theory of the longitudinal vibration of anistropic elastic bars is presented. The equation of motion and the associated boundary conditions are derived by the application of Hamilton's principle. The developed theory is a Rayleigh-Bishop type model of the longitudinal vibration. An analogy is formulated between the longitudinal vibration of an anistropic bar and the bending vibration of an isotropic Euler-Bernoulli-Rayleigh beam subjected to constant axial force.

For an orthotropic elastic bar the connection between the position of material coordinate system, which is given by an angle coordinate, and the eigenfrequencies is 
analyzed. Obtained numerical results for some values of eigenfrequencies by the application of the approximate analytical method are checked by FEM (Abaqus) computations. Presented numerical results show that the eigenfrequencies of the longitudinal free vibrations slightly depend on the position of principal axes of orthotropy.

Acknowledgement. The described study was carried out as part of the EFOP-3.6.1-162016-00011 "Younger and Renewing University - Innovative Knowledge City - institutional development of the University of Miskolc aiming at intelligent specialisation" project implemented in the framework of the Szechenyi 2020 program. The realization of this project is supported by the European Union, co-financed by the European Social Fund and supported by the National Research, Development and Innovation Office - NKFIH, K115701.

\section{REFERENCES}

1. A. E. H. Love. A Treatise on the Mathematical Theory of Elasticity. 4th ed. Dover, New York, 1944. URL: https : //hal . archives-ouvertes.fr/hal$01307751 /$ document.

2. J. W. Rayleigh. Theory of Sound. Dover, New York, 1945.

3. S. P. Timoshenko, D. H. Young, and W. Weaver. Vibration Problems in Engineering. 4th. ed. Wiley, New York, 1974.

4. J. S. Rao. Advanced Theory of Vibration. Wiley Eastern Limited, New Delhi, 1992. URL: https://books.google.hu/books?id=ComyQgAACAAJ.

5. S. S. Rao. Mechanical Vibrations. 4th. ed. Prentice Hall, Upper Saddle River, NJ., 2004.

6. S. S. Rao. Vibration of Continuous System. New Jersy, John Wiley, 2007.

7. R. E. D. Bishop. "Longitudinal waves in beams." Aeronautical Quarterly, 3(2), (1952), pp. 280-293. DOI: 10.1017/S0001925900000706

8. M. Shatalov, I. Fedotov, H. M. Tenkam, and J. Marais. "Comparison of classical and modern theories of longitudinal wave propagation in elastic rods." The Sixteenth International Congress on Sound and Vibration. Krakow. 2009. URL: http://hdl.handle.net/10204/3629

9. I. Fedotov, T. Fedotova, M. Shatalov, and H. M. Tenkam. "Application of eigenfunction orthogonalities to vibration problems." Proceedings ot the World Congress on Engineering. London UK., 2009.

10. M. Shatalov, J. Marais, I. Fedotov, and H. M. Tenkam. "Longitudinal vibration of isotropic solid rods: From classical to modern theories." Advances in Computer Science and Engineering, Ch.10 (2011), pp. 1-28. URL: http://hdl . handle. net/10204/5739.

11. L. Meirovitch. Fundamentals of Vibrations. McGraw-Hill, New York, 2001. URL: https://www . amazon.com/Fundamentals-Vibrations-Leonard-Meirovitch/ dp/1577666917.

12. S. G. Lekhnitskii. Theory of Elasticity of an Anisotropic Body. Mir Publishers, 1981. DOI: $10.1137 / 1009023$.

13. J. D. Renton. Elastic Beams and Frames. Camford Books, Norfolk, 2000.

14. L. Elsgolts. Differential Equations and Calculus of Variations. University Press of the Pacific, 2003. URL: https://books .google.hu/books?id=ZwIyAAAACAAJ. 
15. E. J. R. Charles. Ordinary Differential Equations. Applications, Models and Computing. CRC Press, Boca Ranton, 2010. URL: https://www.crcpress.com/ Ordinary-Differential-Equations-Applications-Models-and-Computing/ Roberts/p/book/9781138118218.

16. L. Meirovitch. Principle and Techniques of Vibrations. Prentice Hall, New Jersey, 1997. URL: https : / / www - pearson . com/us / higher-education/program / Meirovitch-Principles-and-Techniques-of-Vibrations/PGM272917.html.

17. C. T. Herakovich. Mechanics of Fibrous Composites. John Wiley \& Sons, Inc., 1998. DOI: 10.1007/978-94-011-3670-9.

18. H. Altenbach, J. Altenbach, and W. Kissing. Mechanics of Composite Structural Elements. Springer-Verlag Berlin, 2004. DOI: 10.1007/978-3-662-08589-9. 